\title{
Design of a Modular Multilevel Converter as an Active Front-End for a magnet supply application
}

\author{
${ }^{1}$ Panagiotis Asimakopoulos, ${ }^{1}$ Konstantinos Papastergiou, ${ }^{2}$ Massimo Bongiorno \\ ${ }^{2}$ Chalmers University of Technology, Gothenburg, Sweden, ${ }^{1}$ CERN, Geneva, Switzerland \\ Keywords: Multilevel Converters, Active Front-End, Accelerators, Design
}

\begin{abstract}
The aim of this work is to describe the general design procedure of a Modular Multilevel Converter (MMC) applied as an Active Front-End (AFE) for a magnet supply for beam accelerators. The dimensioning criteria for the converter and the dc-link capacitance are presented and the grid transformer requirements are set. Considering the converter design, the arm inductance calculation is based on the specifications for the arm-current ripple and the DC-link fault tolerance, but, also, on the limitation of the second harmonic and the second-order LC resonance of the arm current. The module capacitance value is evaluated by focusing on the required switching dynamics and the capacitorvoltage ripple according to a newly proposed graphical method. The loading of each semiconductor in the half bridge is calculated via simulation, indicating the unsymmetrical current distribution. It is concluded that the current distribution for each semiconductor depends on the mode of operation of the converter. The different criteria for the choice of the number of modules per arm are discussed. Finally, the complete system, including a model for the load and the converter control strategy applied to the converter is simulated and obtained results are presented.
\end{abstract}

Presented at: EPE 2014, 26-28 August 2014, Lappeenranta, Finland

Geneva, Switzerland

January, 2015 


\title{
Design of a Modular Multilevel Converter as an Active Front-End for a magnet supply application
}

\author{
Panagiotis Asimakopoulos, Konstantinos Papastergiou \\ CERN, European Laboratory for Particle Physics \\ CH-1211, Geneva 23, Switzerland \\ Tel.: +41227675183 \\ email: panagiotis.asimakopoulos@cern.ch \\ k.papastergiou@cern.ch \\ URL: http://home.web.cern.ch/
}

\author{
Massimo Bongiorno \\ Chalmers University of Technology \\ SE-412 96, Gothenburg, Sweden \\ Tel.: +46317721631 \\ email: massimo.bongiorno@chalmers.se \\ URL: http://www.chalmers.se
}

\section{Acknowledgements}

This work was elaborated in the Electrical Power Converters group of the European Organization for Nuclear Research (CERN) in collaboration with the Department of Energy and Environment at Chalmers University of Technology, Gothenburg, Sweden.

\section{Keywords}

«Multilevel Converters», «Active Front-End», «Accelerators», «Design»

\begin{abstract}
The aim of this work is to describe the general design procedure of a Modular Multilevel Converter (MMC) applied as an Active Front-End (AFE) for a magnet supply for beam accelerators. The dimensioning criteria for the converter and the dc-link capacitance are presented and the grid transformer requirements are set. Considering the converter design, the arm inductance calculation is based on the specifications for the arm-current ripple and the DC-link fault tolerance, but, also, on the limitation of the second harmonic and the second-order LC resonance of the arm current. The module capacitance value is evaluated by focusing on the required switching dynamics and the capacitorvoltage ripple according to a newly proposed graphical method. The loading of each semiconductor in the half bridge is calculated via simulation, indicating the unsymmetrical current distribution. It is concluded that the current distribution for each semiconductor depends on the mode of operation of the converter. The different criteria for the choice of the number of modules per arm are discussed. Finally, the complete system, including a model for the load and the converter control strategy applied to the converter is simulated and obtained results are presented.
\end{abstract}

\section{Introduction}

The Modular Multilevel Converter (MMC) [7] is a new technology that has already started to be applied in the field of medium and high power electronics. It demonstrates several interesting advantages over other topologies such as:

- the redundancy in operation due to additional modules with the possibility to be inserted or bypassed

- the low switching frequency of the semiconductor devices limiting their needs for cooling, prolonging their lifetime and minimizing their switching losses

- given a sufficient number of modules per arm, that is feasible due to the scalability of the topology, the production of sinusoidal waveforms with very low harmonic content reduce drastically the size of the filters used at the ac interface of the converter. 
The MMC studied in this work is based on the half-bridge module. Several modules connected in series with an inductance form a converter arm, according to Fig. 1. A phase-leg is split in two arms, the upper and the lower one. In an MMC the full dc-link voltage is equally distributed among the modules of an arm. For a dc link voltage $V_{d c}$ and for $N$ modules per arm, the average voltage per module capacitor is $\frac{V_{d c}}{N}$.

Fig. 1: Three-phase MMC circuit

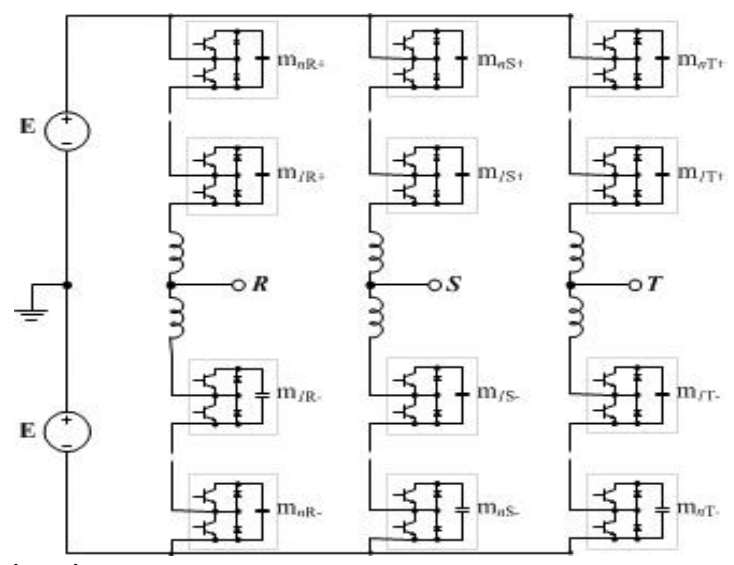

\section{System Description}

In the actual application considered for this work, the magnet supply system consists of a dc-link capacitors bank and an H-bridge converter that regulates the voltage applied to a magnet load of the system, see Fig. 2. The magnet is modeled as an inductive load with a parasitic resistance. An Active Front-End (AFE) adjusts the dc-link voltage and a transformer connects the system to the grid.

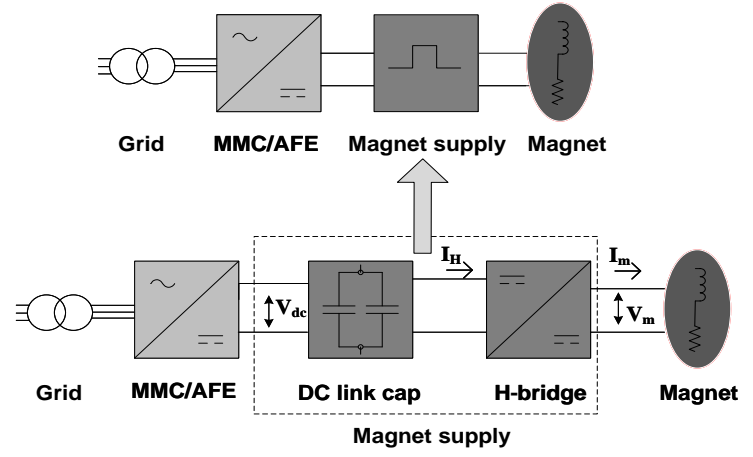

Fig 2: Complete Structure of the magnet supply

\section{Design process}

Based on the load specifications, the design process for the following components of the system described in Fig. 2 will be illustrated:

1. Dimensioning of dc-link capacitance

2. AFE Converter power and components ratings

\section{Load specifications}

The specifications for the magnet are $L_{m}=0.18 H, R_{m}=0.5 \Omega$ and the current waveform applied has trapezoidal shape with a period $T=0.9 \mathrm{~s}$. The magnet current follows the pattern in Table 1 .

Table 1: Magnet current values

\begin{tabular}{|l|l|l|l|l|l|l|}
\hline Time [s] & 0 & 0.1 & 0.5 & 0.53 & 0.7 & 0.9 \\
\hline Current [A] & 400 & 400 & 6000 & 6000 & 400 & 400 \\
\hline
\end{tabular}

\section{Dimensioning of DC-link capacitor bank}


The dc-link capacitor is sized to have enough stored energy to provide the maximum energy specified for the magnet inductance and at the same time to limit the ripple to the specified maximum value of $20 \%$. The maximum magnet voltage during the load supply is calculated to be $6 \mathrm{kV}$. It must be ensured that the lowest capacitor voltage value is higher than the voltage across the load. Therefore, the minimum dc-link capacitor voltage is chosen to be $V_{d c, \min }=6.5 \mathrm{kV}$, in order to provide a voltage margin at the input of the H-bridge for a modulation index lower than 1 . Thus the initial capacitor voltage must be

$$
V_{d c .0}=\frac{V_{d c . \min }}{0.8}=8.125 \mathrm{kV}
$$

The maximum energy is stored in the magnet inductance when the magnet current is maximum as well. Thus,

$$
E_{L \cdot \max }=\frac{1}{2} L_{m} I_{m}^{2}=3.24 M J
$$

The capacitance of the dc-link capacitor can be calculated

$$
E_{C}(t)=E_{L \cdot \max }=\frac{1}{2} C_{d c} V_{d c, 0}^{2}-\frac{1}{2} C_{d c} V_{d c, \min }^{2} \Leftrightarrow C_{d c}=\frac{2 E_{L \cdot \max }}{V_{d c, 0}^{2}-V_{d c, \min }^{2}}=0.273 F
$$

where $E_{C}(t)$ is the capacitor's stored energy. The capacitor's time constant is $\tau_{c}=0.369 \mathrm{~s}$.

\section{Converter power and component ratings}

The converter is going to be designed with the aim to supply the maximum resistive losses of the magnet.

\section{Semiconductor ratings}

The voltage of the module capacitor that is connected in parallel to the semiconductors defines the semiconductors voltage ratings. The average module capacitor voltage is equal to $\frac{V_{d c, 0}}{N}$ and an extra margin is added for over dimensioning, in order to provide the expected voltage plus the ripple at the capacitor. Margins may be added to extend the semiconductor lifetime and fault-proof operation. In this application the absolute essential ratings are calculated.

The current loading is not the same for all the semiconductors of the module. They vary depending on the mode of operation of the converter.

The current in each arm of the converter is expressed by:

$$
i_{u / l, j}=\frac{i_{d c}}{3} \pm \frac{\hat{i_{1}}}{3}
$$

where the subscript $\mathrm{u} / \mathrm{l}$ indicates if the upper or the lower arm of the converter is considered, while $\mathrm{j}$ denotes the respective phase leg; $i_{d c}$ is the dc component and $i_{1}$ is the fundamental frequency component of the current.

The converter can operate either in inverter or rectifier mode. In the rectifier mode the dc component of the arm current is considered to be negative and in the inverter mode positive, based on the real power flow sign convention. As a result an offset is added to the arm current; see (1) and Fig.3. In the rectifier mode the negative offset results to higher stress for the lower diode and the upper switch.

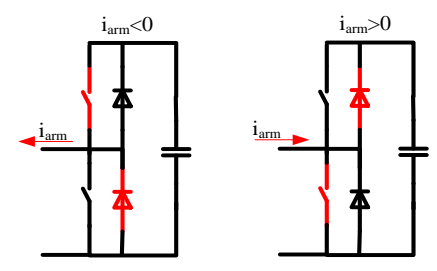

Fig.3: Load for each semiconductor depending on the current sign 
Observing Table 3 the difference between the rms and the peak current indicates that the repetitive current peak of the device has to be considered at the selection process.

Table 3: Semiconductors rms and peak current values

\begin{tabular}{|l|l|l|}
\hline Semiconductor & rms (A) & peak (A) \\
\hline Upper switch & 490 & 2795 \\
\hline Lower switch & 203 & 1028 \\
\hline Upper diode & 357 & 1028 \\
\hline Lower diode & 1482 & 2795 \\
\hline
\end{tabular}

In Fig. 4 the current waveforms for the switches and the arm current are depicted on the left and on the right for the diodes and the arm current again.
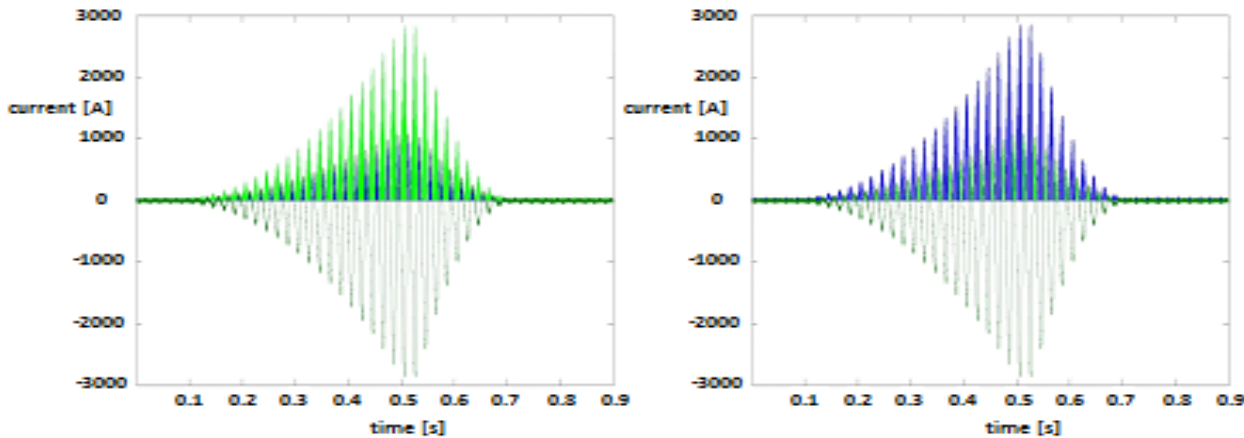

Fig.4: On the left: upper switch (light green), lower switch (blue), arm current (dark green) On the right: upper diode (light green), lower diode (blue), arm current (dark green)

\section{Module capacitance}

The value of the module capacitance influences the ripple at the capacitor voltage. The voltage ripple is in its turn crucial for the lifetime of the capacitor and the output voltage of the converter. The capacitance value has also an impact on the system speed of response. Furthermore, an increase in the capacitance leads to a bigger physical size of the capacitors, affecting the total cost of the construction. Due to the fact that the capacitors are a considerable part of the total system cost, it is important to specify accurately enough the capacitance value for the modules.

In every fundamental cycle, electrical charge is shifted in and out of the module capacitor. This causes a voltage ripple across the capacitor.

Based on the voltage reference for the upper arm, it is expected that the upper capacitor starts to be inserted, in order to achieve the negative load voltage. The discharge part of the capacitor's curve is made with almost continuous insertion of the module and it can be assumed continuous between the two red lines in the upper module output voltage plot, Fig. 5. The upper arm current is assumed to be sinusoidal with frequency equal to the fundamental frequency and it will be negative during the capacitor discharge. It can be described by the equation

$$
i_{u}=i_{c}+\frac{\hat{i}_{1}}{2}
$$

The aim is to calculate the capacitor size for a given ripple and load. According to the equation

$$
C_{\mathrm{mod}}=\frac{i_{c} d t}{d V_{c}}
$$

where is $\mathrm{C}_{\text {mod }}$ the capacitance, $i_{c}$ is the capacitor current that is equal to the upper arm current, if the capacitor is inserted, $d t$ the time that the capacitor discharge lasts and $d V_{c}$ the desired capacitor ripple. By equating the dc-link power provided and the load power, assuming again a lossless system and a modulation index equal to 1 


$$
V_{d c} i_{d c}=V_{0, m s} i_{1, m s} \Leftrightarrow V_{d c} i_{d c}=\frac{\hat{V_{0}} \hat{i}_{1}}{2} \Leftrightarrow \hat{i_{1}}=\frac{2 V_{d c} i_{d k}}{\hat{V}_{0}} \Leftrightarrow \hat{i_{1}}=4 i_{d c}
$$

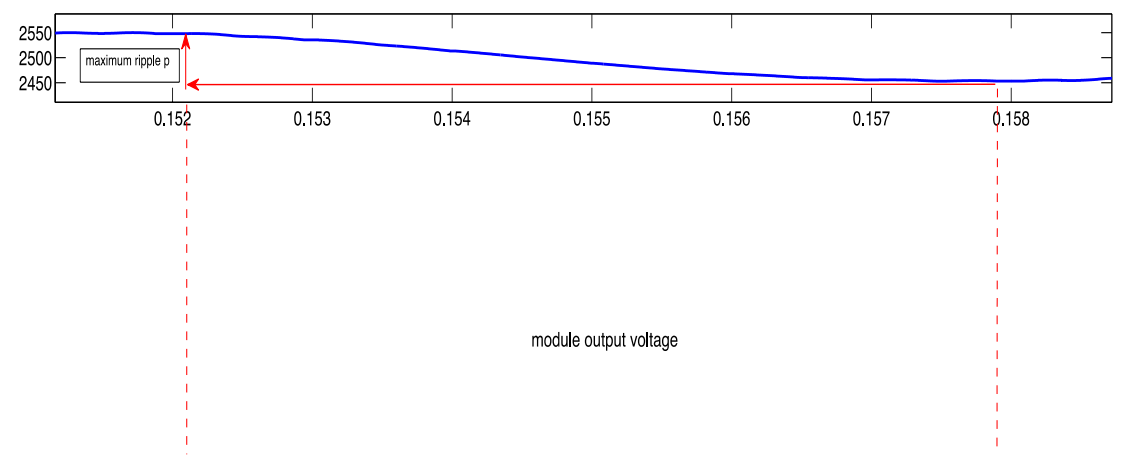

Fig. 5: Upper capacitor voltage ripple, upper arm current and upper module output voltage

The relation between the load current and the dc current is independent of the number of modules. The duration during which the upper arm current is negative is found by solving the equation

$$
i_{d c}-\frac{\hat{i_{1}}}{2} \sin (\omega t)=0 \Leftarrow(6) \Rightarrow \sin (\omega t)=\frac{1}{2}
$$

This equation has two solutions for $\omega t=\frac{\pi}{6}$ or $\omega t=\frac{5 \pi}{6}$ that correspond to the beginning and end of the discharging time respectively.

The charge lost at the capacitor during its energy extraction is

$$
Q_{C}=\int_{t_{1}}^{t_{2}} i_{C} d t=\int_{t_{1}}^{t_{2}}\left[i_{d c}-\frac{\hat{i_{1}}}{2} \sin \left(\omega_{1} t\right)\right] d t
$$

where $t_{1}$ is the time instant that corresponds to the first (beginning of discharging) and $t_{2}$ to the second solution (end of discharging) of (7).

Following the same process for a three-phase system by equating the dc-link power provided and the load power, $Q_{c}$ can be written solving the integral in (8).

$$
Q_{C}=\frac{i_{d c}\left(t_{2}-t_{1}\right)}{3}+\frac{\hat{i_{1}}}{2 \omega_{1}}\left(\cos \left(\omega_{1} t_{2}\right)-\cos \left(\omega_{1} t_{1}\right)\right)
$$

where the dc component of the current in every leg is $\frac{i_{d c}}{3}$.

By substituting $i_{c} d t$ with $Q_{c}$ in (9) for a specified voltage ripple $d V_{c}$, the capacitance needed can be found.

$$
C_{\text {mod }}=\frac{\frac{i_{d c}\left(t_{2}-t_{1}\right)}{3}+\frac{\hat{i}_{1}}{2 \omega_{1}}\left(\cos \left(\omega_{1} t_{2}\right)-\cos \left(\omega_{1} t_{1}\right)\right)}{d V_{C}}
$$

The method is valid for a high number of modules keeping the $f_{s w}$ fixed. The time interval, where the capacitor is discharged, depends on the fundamental frequency but the method is the same based on the interval where the arm current is negative with respect to the fundamental period. If $N$ is increased the peak-to-peak voltage remains almost the same, see Fig. 6, where the upper capacitor voltage value is shown for $N=2,4,6,10,15$ and for the same value of $C_{\text {mod }}, L_{\text {arm }}$ and $f_{\text {sw }}$.

The small difference in the voltage ripple can be explained by the fact that more capacitors are inserted or bypassed in the arm during the discharge of a capacitor, thus influencing the upper arm current.

While $N$ increases, the ripple in the capacitor voltage tends to be constant due to the fact that the ripple 
in the arm current is eliminated. In addition, the carriers are shifted to the reference with an angle that depends on $N$, due to the fact that a separate carrier is used for each module and is compared to a general upper arm voltage reference. This phase shift can affect the capacitor-voltage ripple if a quite low $f_{s w}$ is used.

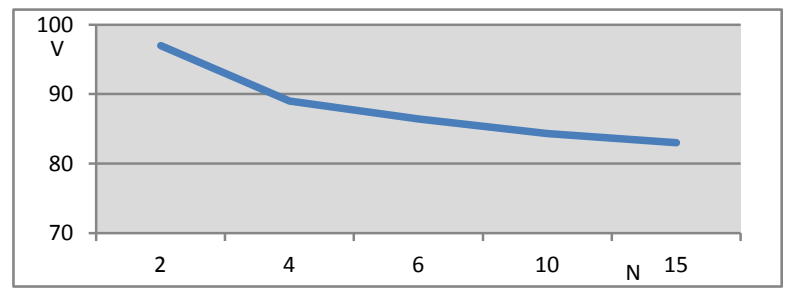

Fig. 6: Capacitor voltage ripple as a function of number of modules per arm

\section{Design of the arm inductance}

The arm inductance can be dimensioned based on the following criteria:

1. Current ripple

2. Limitation of the second harmonic component in the arm current

3. Limitation of fault current at the dc-link

4. $\mathrm{L}_{\text {arm }}$ and $\mathrm{C}_{\text {mod }}$ resonance peak limitation

1. Current ripple

The first criterion is the ripple in the load current. The ripple in the arm current and, as a consequence, in the load current is inversely proportional to the number of modules per arm $N$. With an increasing $N$ the equivalent switching frequency increases and the time interval that each module is inserted reduces. Therefore, the voltage difference between the dc-link side and the sum of the modules is applied to the arm inductance for a smaller period of time and the current ripple is limited

$$
d i=\frac{V_{L} d t}{L_{a r m}}
$$

where $\mathrm{dt}$ is the time interval, $\mathrm{V}_{\mathrm{L}}$ the voltage applied to the arm inductance and $\mathrm{i}$ the arm current, either for upper or lower arm. From Fig. 7 it is possible to observe that an increased number of modules $\mathrm{N}$ results in a reduced ripple in the current. In Fig. 7 the same value for the arm inductance has been used independently of the number of modules $N$ at a single-phase MMC to demonstrate the inductance effect on the arm-current ripple.

2. Second harmonic component in the arm current

The second criterion for the design of the arm inductance is the limitation of the second harmonic component of the current circulating among the phase legs. This can be achieved by a passive filter [4] or a dedicated controller [6].

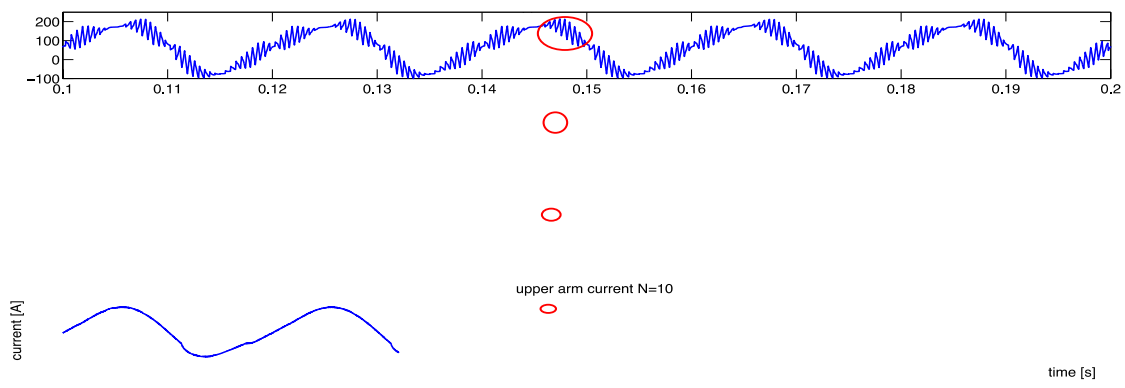

Fig. 7: Arm current for different number of modules per arm, from top to bottom: N=2, 4, 6, 10

\section{Limitation of fault current at the dc-link}

The third criterion ensures that the current value in case of a short circuit at the dc-link would not exceed the current limitation of the antiparallel diodes within the time period that the protection equipment needs to react. The half-bridge module is by-passed via the antiparallel diodes and no protection is provided. If a large enough arm inductance is selected, it may result to a high voltage 
drop especially for high current applications. Another solution is to use a full-bridge module, where the current is controlled in both directions.

Figure 8 illustrates the current and voltage during the fault in the case of the half-bridge module MMC. The voltage disturbance occurs at $\mathrm{t}=0.6 \mathrm{~s}$ and it is assumed that it is detected and cleared at $\mathrm{t}=0.64 \mathrm{~s}$. The fast current rise and its high value are an indication that a large arm inductance value is needed.

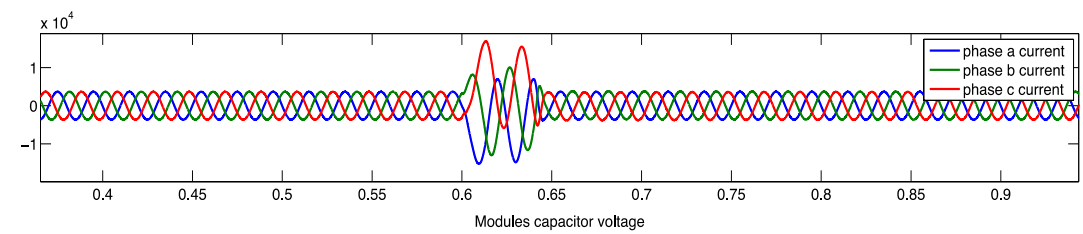

$\sum_{0}$
兽

Fig. 8: From top to bottom: Phase currents and modules capacitor voltages for half-bridge MMC

In case of high current applications, considerable voltage drop occurs across a large arm inductance. Therefore, either the voltage must be increased or the full bridge module can be used.

In Fig. 9 the same fault for a full bridge MMC is simulated. The module capacitors are connected in series opposing the current to rise. The current is kept at low levels during the fault but it must be ensured that the capacitors can handle such a voltage fluctuation. The arm inductance can be selected now based on the capacitor voltage fluctuation.

For the full-bridge module extra considerations are needed for the capacitors ripple tolerance.

4. Larm and Cmod resonance peak limitation

Finally, to avoid the resonant peak created by the components $L_{\text {arm }}$ and $C_{m o d}$ in a frequency double of the fundamental the following equation must be satisfied [5]

$$
L_{\text {arm }}>\frac{5 N}{12 \omega^{2} C_{\text {mod }}}
$$

Therefore, for a given value of the module capacitance, the arm inductance value must be ensured to fulfill (12).

The criteria that are actually applied in this work are the current ripple and the $\mathrm{L}_{\text {arm }}, \mathrm{C}_{\text {mod }}$ resonance peak limitation.

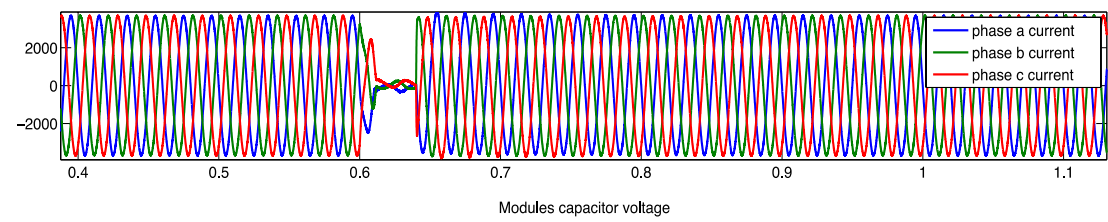

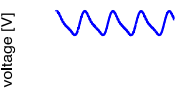

$$
\text { time }[s]
$$

Fig. 9: From top to bottom: Phase currents and modules capacitor voltages for full-bridge MMC

\section{Selection of the number of modules per arm}

The selection for the number of modules per arm $N$ is based on the following criteria:

- The voltage ratings of the semiconductors; as it is observed the module semiconductor rating is related to the equation $\frac{V_{d c}}{N}$. 
- $\quad$ The same ratings are valid for the module capacitors. Assuming that $N$ is doubled, the module capacitance will be doubled; on the other hand, the stored energy will be half, as it can be seen from

$$
C_{\text {mod }}=\frac{Q_{C}}{0.2 \frac{V_{d c}}{N}} \text { and } W_{C}=\frac{1}{2} C u_{c}^{2}
$$

- The grid-current ripple; if the number of modules is large, the voltage step between the modules insertions/bypasses is small, the equivalent switching frequency is high and, therefore, the current ripple is small. As a result, the arm inductance value can be reduced.

Table 2 shows the ratings of the simulated converter. The ohmic losses at the load reach $18 \mathrm{MW}$ that is the power rating of the converter. The converter is selected to have four modules per arm.

Table 2: Converter ratings

\begin{tabular}{|l|l|}
\hline Power rating (MVA) & 18 \\
\hline DC-link voltage (V) & 8125 \\
\hline Line to Line rms voltage (V) & 4976 \\
\hline Number of modules per arm N & 4 \\
\hline Module capacitance (mF) & 10 \\
\hline Module capacitor average voltage (V) & 2031.25 \\
\hline Maximum arm rms current (A) & 2230 \\
\hline Arm inductance (mH) & 1.7 \\
\hline Switching frequency (Hz) & 1000 \\
\hline Equivalent switching frequency (Hz) & 8000 \\
\hline
\end{tabular}

\section{Transformer requirements}

The transformer that connects the medium voltage grid of $18 \mathrm{kV}$ with the converter should be selected for a voltage ratio of $18 \mathrm{kV} / 3.8 \mathrm{kV}$. The leakage inductance $L_{l k}$ can be found

$$
L_{l k}=\frac{V_{\text {phase } r m s} K_{c c}}{\omega_{1} I_{\text {phase,rms }}}
$$

where $V_{\text {phase,rms }}$ the rms phase voltage at the secondary winding of the transformer, $I_{\text {phase,rms }}$ the secondary winding current, $\omega_{1}$ the fundamental angular frequency of the grid and $K_{c c}$ the short-circuit impedance of the transformer with a typical value between $6 \%$ and $10 \%$. The leakage inductance contributes to the current filtering and the dc-link fault current limitation.

\section{Load model}

Due to the fact that the load seen by the converter dc-link is the input of the H-bridge, see Fig. 2, a more detailed load model representing the total system of the H-bridge connected to the magnet is designed. By equating the input power of the H-bridge with the power drawn by the magnet, the current supplied at the H-bridge input can be calculated by

$$
\eta P_{H}=P_{m} \Leftrightarrow \eta V_{d c} I_{H}=V_{m} I_{m} \Leftrightarrow I_{H}=\frac{V_{m} I_{m}}{\eta V_{d c}}
$$

where $\eta$ is the H-bridge efficiency, while $V_{m}$ and $I_{m}$ are the magnet voltage and current, respectively. The H-bridge is considered to be lossless, with efficiency equal to 1 . The H-bridge input current is represented as the output of a controlled current source. The magnet current is predefined, see Table 1 , and used to calculate the voltage drop $V_{m}$ across the inductance and the resistance of the magnet. 


\section{Control strategy}

The control of the MMC as an AFE consists of two levels, see Fig. 10. The first level concerns the inner balancing of the capacitors' voltage level and the dc-link voltage control. It includes the individual balancing control for every module and the average balancing control for the average voltage level of each phase-leg [9]. The applied modulation technique is the Phase-Shifted carrier Pulsed Width Modulation (PS-PWM).

The second level of control is an ac current controller and takes care of the connection of the system with the grid. Due to the fact that the converter supplies the real power losses of the load, the active component of the current reference is equal to the active component of the current needed for the power losses of the load. The reactive component of the current is zero.

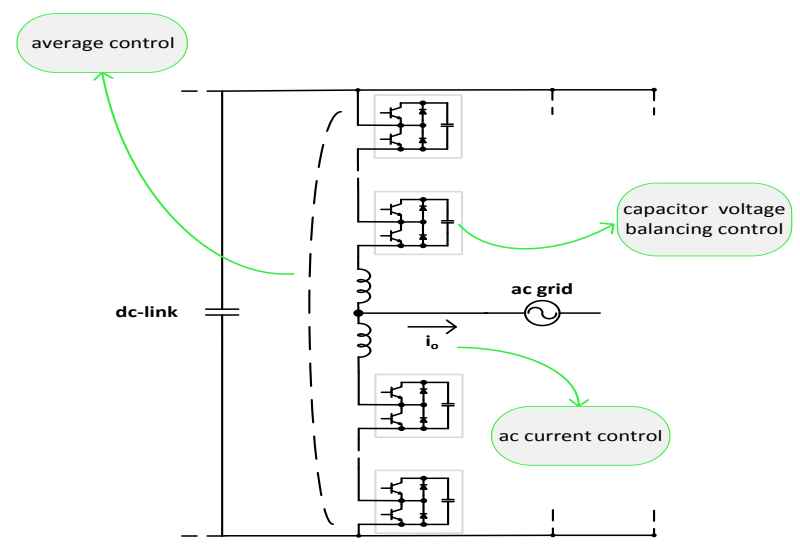

Fig. 10: MMC control overview

The controller is implemented in the synchronously rotating dq reference frame, where the d-axis is aligned with the voltage vector. As a result, the $d$ component of the current takes over the active power exchange and the q component the reactive power. Fig. 11 shows the simulation results of the system based on the MMC with the ratings of Table 2. The MMC is the AFE and the load model described above replaces the H-bridge and the magnet.
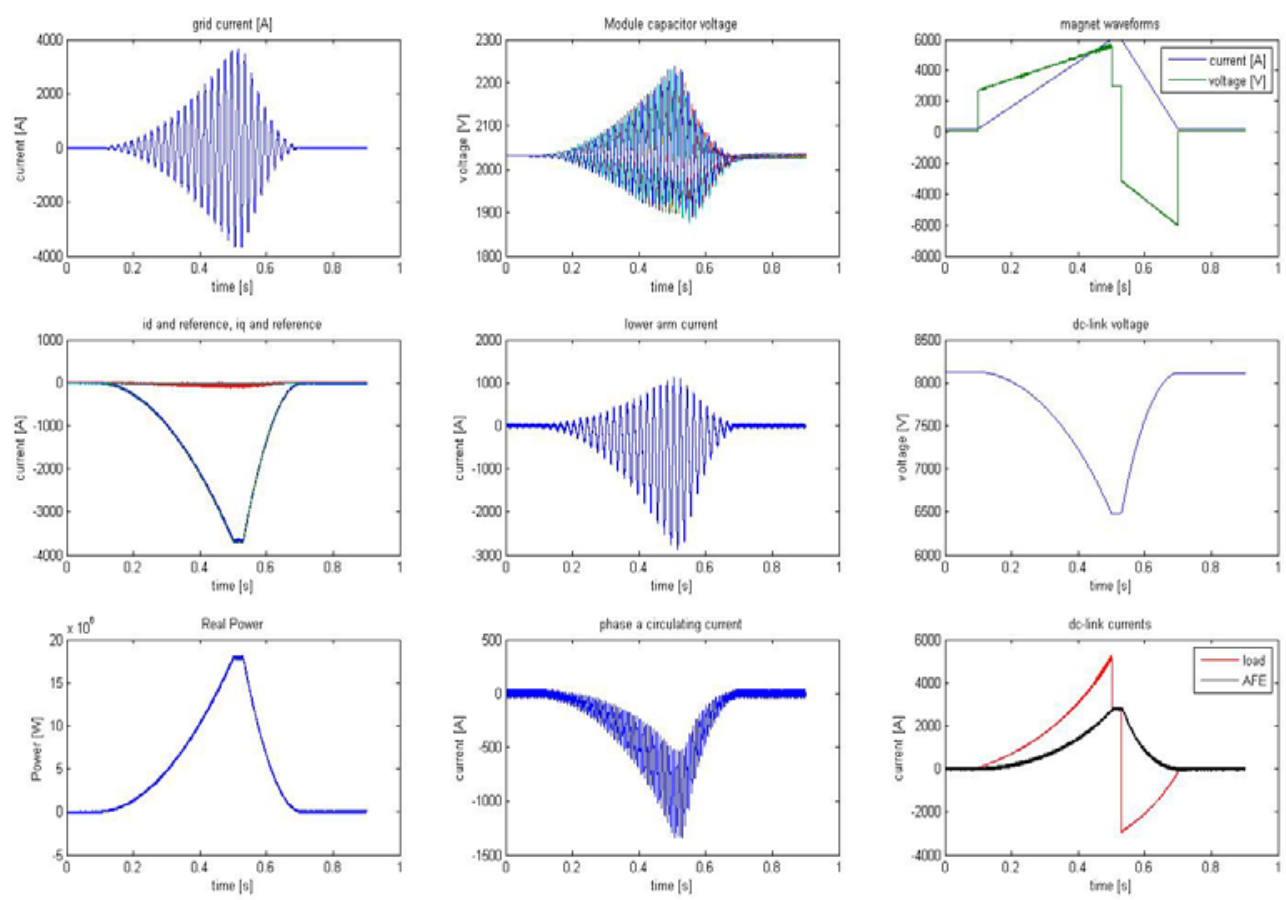

Fig. 11: Simulation results overview for the system with MMC as an AFE 
Focusing on the most important information, at the left plot of the first row the grid current reaches a maximum peak value of approximately $3.7 \mathrm{kA}$ at $\mathrm{t}=0.5 \mathrm{~s}$. The Total Harmonic Distortion (THD) of the current is equal to $0.78 \%$. It is calculated at the maximum value of the current waveform between $\mathrm{t}=0.5 \mathrm{~s}$ and $\mathrm{t}=0.53 \mathrm{~s}$, because the load at this interval is constant and the system is at steady state. At the second plot of the second row the capacitors voltage ripple is maximum at the peak load and equal to $17 \%$ of the average capacitor voltage. The module capacitance was designed for a maximum voltage ripple of $20 \%$ at the peak load. The difference between the theoretical calculations and the simulation occurs because it was assumed that no second harmonic exists in the arm current. At the first plot of the second row it is observed that the components of the grid current, real component $i_{d}$ in blue and reactive $i_{q}$ in red, are decoupled and they track successfully their references. At the second plot of the second row the lower arm current consists mainly of the dc component and the first harmonic. It has a negative dc offset because the real power flow is towards the dc-link side. The dc-link voltage decreases to $6.5 \mathrm{kV}$ as expected and returns again to its initial value.

\section{Conclusions}

This paper provides an overall guideline for the dimensioning of the MMC as an AFE, in this case applied to beam accelerator applications. The module capacitance is of great importance for the total cost, the size and the response of the system. The capacitance for a specified ripple is calculated using the ac and dc side currents of the converter. The calculations can be adapted to any fundamental frequency and are independent of the switching frequency. Moreover, it is observed that the load is not distributed equally among the semiconductors. The factor, which defines which devices are most loaded, is the mode of the converter operation. In the case of the rectifier mode, the upper switch and the lower diode are the most loaded components. The main arm inductance design criteria are the current ripple that can be reduced by increasing $\mathrm{N}$, the second harmonic component that can be suppressed by using a passive filter or a controller and, finally, the dc-link fault current limitation. The dc-link current can be limited either by increasing the arm inductance value to decrease the current rise rate or by replacing the half-bridge with the full-bridge module. In the case of the full-bridge module the capacitors' ripple tolerance should be considered. The decision for the number of $\mathrm{N}$ is a matter of techno-economical nature. The above conclusions have been confirmed by simulation.

\section{References}

[1] A. Antonopoulos, L. Angquist, H.-P. Nee, “On Dynamics and Voltage Control of the Modular Multilevel Converter”, European Power Electronics Conference (EPE), Barcelona, Spain, September 8-10, 2009.

[2] Q. Tu, Z. Xu, H. Huang and J. Zhang, "Parameter Design Principle of the Arm Indcutor in Modular Multilevel Converter based HVDC”, International Conference on Power System Technology, 2010.

[3] D. Schmitt, Y. Wang, Th. Weyh and R. Marquardt, "DC-side Fault Current Management in extended Multiterminal-HVDC-Grids”, $9^{\text {th }}$ International Multi-Conference on Systems, Signals and Devices, 2012.

[4] K. Ilves, S. Norrga, L. Harnefors and H.-P. Nee, “Analysis of Arm Current Harmonics in Modular Multilevel Converters with Main-Circuit Filters”, $9^{\text {th }}$ International Multi-Conference on Systems, Signals and Devices.

[5] K. Ilves, "Modelling and Design of Modular Multilevel Converters for Grid Applications”, Licentiate Thesis, Stockhom, Sweden, 2012.

[6] X. She, A. Huang, X. Ni and R. Burgos, "AC Circulating Currents Suppression in Modular Multilevel Converter”, 38 ${ }^{\text {th }}$ Annual Conference on IEEE Industrial Electronics Society, Montreal, Canada, 2012.

[7] A. Lesnicar, R. Marquardt, “A new modular voltage source inverter topology”, EPE, Toulouse, France, 2003.

[8] M. Saeedifard, R. Iravani, "Dynamic Performance of a Modular Multilevel Back-to-Back HVDC System, IEEE TRANSACTIONS ON POWER DELIVERY, VOL. 25, NO. 4, October, 2010.

[9] M. Hagiwara, H. Akagi, "Control and Experiment of Pulsewidth-Modulated Modular Multilevel Converters”, IEEE TRANSACTIONS ON POWER ELECTRONICS, VOL. 24, NO. 7, JULY, 2009.

[10] S. Rohner. S.Bernet, M. Hiller and R. Sommer, "Modelling, Simulation and Analysis of a Modular Multilevel Converter for Medium Voltage Applications”, IEEE International Conference on Industrial Technology, 14-17 March, 2010. 